\title{
RECHERCHES SUR LA MAMMITE DE LA VACHE
}

par

\section{R. N. GORANSSON}

Médecin-Vétérinaire des Services d'Inspection des Denrées alimentaires de la ville d'Anvers.

Au cours des années 1929 et 1930, lors de l'examen microscopique du lait de 28 vaches atteintes de mammite, je constatai la présence, dans tous les échantillons, de microcoques prenant le Gram, isolés, en diplocoques ou en grappes; associés, seulement 5 fois, à des streptocoques prenant le Gram. A la même époque, l'examen microscopique de frottis de matériel provenant de mamelles, présentant des lésions de mammite, de vaches abattues à l'abattoir d'Anvers, permit de constater, dans 90 sur 92 cas, la présence de microcoques similaires, associés seulement 36 fois à diautres microorganismes, parmi lesquels je ne trouvai que 17 fois des streptocoques se colorant ou non par le Gram [1].

Lors de la publication de ces données, j'émis l'avis que, par l'ensemencement du matériel prélevé dans des milieux de culture, la présence de streptocoques aurait probablement été constatée dans un plus grand nombre de eas. Les données trouvées dans la littérature, entre autres celles de Gorini [2] et [3], Hucker et Pederson [4], Hucker [5], Kufferath [6], Venibafis [7], JöRGRNSEN-[8], SKAR [9], justifient cet avis.

En ce qui concerne la présence de streptocoques dans le lait et leur forme, considérées comme indications formelles de l'existence de la mammite chez les vaches productrices, si RuHмEкоRF est d'avis qu'on peut distinguer, par la forme de palissade et par la présence d'une capsule entourant parfois la chaînette, le streptocoque pathogène du streptocoque saprophyte [10], OrLA-JENSEN [11], VAN OYEN [12] déclarent que la forme des streptocoques ne donne aucune indication sur leur faculté pathogène. Ces deux auteurs sont encore d'avis que pour le diagnostic de la mammite, il faut recourir à l'examen de laits individuels. VEenbaes [13] est du même avis [13], mais affirme qu'il faut rechercher spécialement les streptocoques par la coloration du Gram, que ces bactéries sont pathogènes pour l'homme et les animaux [14].

Ultérieurement j'eus encore l'occasion d'examiner du matériel, provenant de 85 vaches atteintes de mammite, abattues à l'abattoir d'Anvers, ainsi que six échantillons de lait, provenant de vaches atteintes de mammite, hébergées dans des étables productrices de lait pour la consommation, situées sur le territoire de la ville d'Anvers.

Pour 57 cas, je me limitai à l'examen microscopique de frottis de 
matériel prélevé de la mamelle malade, et chaque fois je constatai la présence de microcoques prenant le Gram, isolés, en diplocoques ou en grappes, associés trois fois à des streptocoques prenant le Gram, trois fois à des streptocoques ne prenant pas le Gram.

En dehors de cela furent encore trouvés : quatre fois des organismes cocciformes isolés ou accouplés ne prenant pas le Gram, douze fois des bacilles prenant le Gram, huit fois des bacilles ne prenant pas le Gram, et, quoique dans cinquante cas les mamelles provenaient de vaches atteintes de tuberculose, seulement douze fois le bacille de Косн. Pour les autres cas, en même temps que l'examen des frottis, du matériel prélevé de la mamelle fut ensemencé dans des échantillons de $10 \mathrm{~cm}^{3}$ de lait stérilisé dans des flacons fermés au moyen de bouchons de caoutchouc. Au moment de l'ensemencement, le lait fut chauffé à la température de $38^{\circ} \mathrm{C}$., de façon à lui donner approximativement la température qu'il a, lorsqu'il sort de la mamelle. Gorisi signale que les bactéries du lait agissent à la fois sur la caséine et sur le lactose ; qu'une température élevée favorise la saccharolyse, une température basse la easéoJyse [15]. Les cultures furent conservées à la température ordinaire, mais par intermittences, pendant une à deux heures, elles furent placées à la température de $38^{\circ} 5 \mathrm{C}$, au cours de périodes d'observation qui, pour dix-neuf cas, varièrent de trois à quatorze jours et qui, pour neuf cas, furent supérieurs à quinze jours. Pendant les périodes d'observation, presque chaque jour fut fait l'examen microscopique de frottis du lait et furent observées non seulement les variations de la flore bactérienne, mais encore les modifications d'aspect de la culture.

Dans les dix-neuf mamelles, pour lesquelles la période d'observation varia de trois à quatorze jours, furent toujours trouvés des microeoques prenant le Gram, isolés, en diplocoques ou en grappes de diverses dimensions, soit seuls, soit associés à d'autres formes bactériennes; des cocei isolés ou en diplocoques ne prenant pas le Gram, des bacilles de différentes dimensions prenant ou ne prenant pas le Gram; une fois, des streptocoques n'ayant pas la forme de palissade et prenant le Gram ; deux fois, des streptocoques n'ayant pas la forme de palissade et ne prenant pas le Gram, et, bien que toutes les mamelles provinssent de vaches atteintes de tuberculose, seulement trois fois le bacille de Косн.

Dans tous les échantillons de lait stérilisé, ensemencé de matériel provenant de ces mamelles, il y eut formation d'un coagulum floconneux homogène, parfois le lendemain, généralement du troisième au sixième jour après l'ensemencement, parfois plus tard, coagulum qui présenta généralement un début de solution lorsque la période d'abservation fut plus longue que cinq jours. 
Dans tous les cas, hormis un seul, pour lequel la période d'observation ne fut que de trois jours, en même temps que se fit la coagulation du lait, ou immédiatement auparavant, apparurent des streptocoques, parfois à courtes, parfois à longues chaînes, présentant ou ne présentant pas la forme de palissade, prenant ou ne prenant pas le Gram. Toujours, lorsqu'il y eut apparition de streptocoques, il y eut des bacilles, parfois longs, parfois courts, parfois à contours ondulés, présentant parfois un étranglement au milieu, prenant ou ne prenant pas le Gram ; parfois à granulations séparées par d'étroits interstices ne prenant pas le colorant ; aspect qui aurait pu justifier l'hypothèse d'un début de scission en unités cocciformes. Ces bacilles apparurent ou devinrent plus nombreux avant l'apparition des streptocoques et présentèrent généralement les mêmes caractéristiques de coloration qu'eux. Ces bacilles devinrent moins nombreux ou disparurent pendant la présence des chaînettes streptococciques et lorsque la période d'observation fut assez longue, celles-ci disparurent et leur disparition coïncida avee l'apparition, dans la culture, d'un plus grand nombre de microcoques isolés et en diplocoyues, présentant les mêmes caractéristiques de coloration que les streptocoques. Lorsqu'il y eut des bacilles tuberculeux dans le frottis de matériel de la mamelle, le lendemain de l'inoculation ces bacilles ne furent pas trouvés dans le lait, qui se coagula en fins flocons le troisième jour. La disparition du bacille de KосH coïncida avec le développement des organismes lactiques, qui précéda la coagulation du lait.

Dans tous les cas, il y eut persistance des microcogues prenant le Gram, isolés, en diplocoques ou en grappes, durant toute la période d'observation.

Pour les neuf autres cas, la période d'observation fut supérieure à quinze jours. Toujours fut observée une évolution à peu près similaire à celle déjà décrite, tant pour l'aspect de la culture que pour la flore bactérienne. Il y eut apparition, disparition, réapparition de streptocoques prenant ou ne prenant pas le Gram, ayant ou n'ayant pas la forme de palissade, dont l'aspect se modifia au cours de la période d'observation; de bacilles prenant ou ne prenant pas le Gram, à contours parfois ondulés et à granulations ; il y eut formation d'un coagulum floconneux dans le lait, solution plus ou moins complète de ce coagulum, pendant que se développa une odeur désagréable, similaire à celle de fromage à la crème très avancé. A la fin de la période d'observation, se trouva généralement dans la culture un précipité plus ou moins abondant de flocons blancs ou jaunâtres, plus ou moins visqueux, au-dessus duquel était un sérum généralement transparent incolore ou jaunâtre, parfois trouble blanchâtre ou jaunâtre. L'aspect des cultures rappelait celui des sérosités qu'on trouve souvent dans 
des mamelles présentant des lésions de mammite chronique, dont d'ailleurs la flore bactérienne ressemble fréquemment à celle de ces cultures : microcoques prenant le Gram, isolés, en diplocoques ou en grappes, bacilles prenant ou ne prenant pas le Gram, longs de 1-1,5 $\mu$, larges de 0,5-06 $\mu$, à extrémités arrondies ou effilées, parfois incurvés, parfois à granulations multiples ou simplement aux extrémités.

Dans un de ces cas il y eut une formation de pigment. Dans lé frottis de matériel de la mamelle, furent trouvés, en dehors des microcoques prenant le Gram, isolés, en diplocoques ou en grappes, des streptocoques prenant le Gram, ayant la forme de palissade, composés de plus de douze unités. Si l'évolution de la flore bactérienne fut similaire à celle des autres cas, il n'en fut pas de même des modifications de l'aspect de la culture. Quelques jours après la coagulation qui eut lieu le sixième jour, l'aspect de la culture se modifia rapidement, sa coloration devint plus foncée et le quarante-deuxième jour, le précipité avait une coloration rouge brunâtre, tandis que dans le sérum trouble jaune foncé flottaient quelques flocons visqueux de même couleur A ce moment, la flore bactérienne était composée de microcoques prenant le Gram, isolés, en diplocoques ou en grappes et de bacilles prenant ou ne prenant pas le Gram, longs de 1-1,5 $\mu$, larges de $0,5-0,6 \mu$, a extrémités arrondies ou effilées, présentant une coloration plus intense aux extrémités.

Dans une de ces mamelles furent trouvés des bacilles tuberculeux. Le second jour, ils furent très abondants dans le lait stérilisé, mais ils disparurent lorsque se développèrent les streptocoques et lorsque se fit la coagulation du lait, indication que le bacille de $\mathrm{KoCH}$ est détruit à un eertain degré d'acidité.

Dans ces eas, les streptocoques trouvés dans les mamelles, présentant des lésions de mammite et ceux apparus dans le lait ensemencé de matériel de ces mamelles, ne présentèrent pas toujours la forme de palissade et ne prirent pas toujours le Gram, bien qu'on puisse supposer, à juste titre, qu'ils étaient pathogènes.

Les six échantillons de lait, qui furent examinés, provenaient de vaches hébergées dans des étables productrices de lait pour la consommation. Ces vaches paraissaient saines et, en dehors d'une, dont le quartier antérieur droit de la mamelle était atrophié et ne donnait plus de lait, tandis que les autres quartiers, dont un seulement donnait du lait à réaction alcaline, ne présentaient pas de lésions décelables à l'examen clinique, aucune d'elles ne présentait une anomalie apparente de la mamelle, en dehors de l'hypertrophie des ganglions rétromammaires.

Tous ces échantillons présentaient une réaction nettement alcaline à l'alizarol, mais donnaient dans le tube de Trommsdorf fes 
sédiments suivants : $0 \mathrm{~cm}^{3} 002,0 \mathrm{~cm}^{3} 008,0 \mathrm{~cm}^{3} 008,0 \mathrm{~cm}^{3} 009$, $0 \mathrm{~cm}^{3} 008,0 \mathrm{~cm}^{3} 018$.

Le premier jour, tout au plus deux heures après le prélèvement des échantillons, l'examen microscopique des frottis de lait révéla seulement la présence de microcoques prenant le Gram, isolés, en diplocoques et en grappes, disposés, dans un seul cas, en conglomérats de grande dimension et formant également de rares chaînettes de trois à quatre unités, n'ayant pas la forme de palissade.

J'eus recours au mềme procédé que celui pratiqué pour les cultures de matériel prélevé sur les mamelles. Les périodes d'observation, au cours desquelles des frottis du lait furent examinés, pour ainsi dire, chaque jour, varièrent de 37 à 64 jours; mais ensuite les échantillons furent laissés à la température ordinaire pendant 25 jours sans être examinés, puis fut fait un dernier examen.

Dans les six échantillons de lait se formèrent des flocons de différentes dimensions, blanes ou jaunâtres, en suspension dans le sérum, du troisième au sixième jour. Au repos ces flocons se déposèrent et au-dessus du précipité se trouva alors un sérum plus ou moins trouble, d'abord blanc bleuâtre, à la superficie duquel se forma une mince couche blanche ou jaunâtre de flocons impalpables, formant voile. Le précipité diminua, pendant que le voile devint plus épais, et que le sérum devint limpide, parfois incolore, parfois jaunâtre. Dans tous les échantillons se développa une odeur désagréable, rappelant celle de fromage à la crème très avancé.

Dans un échantillon, la couleur du voile à la surface du sérum était jaune le quinzième jour ; elle devint plus foncée, vira à l'orange. Vers le quarante-quatrième jour, des granulations rougeâtres s'étaient formées; elles étaient devenues rouges brunâtres le soixante-quatrième jour et avaient la même couleur 25 jours plus tard.

Dans tous les échantillons, avant la coagulation, apparurent des streptocoques prenant le Gram et ayant parfois plus de trente unités, mais ayant seulement quatre fois la forme de palissade. Toujours l'apparition de bacilles précéda la première apparition des streptocoques ou se fit en même temps qu'elle. Parfois ces bacilles prenaient le Gram, étaient remplacés ultérieurement par des bacilles ne prenant pas le Gram ; parfois ils étaient incurvés, certains avaient des contours ondulés, parfois ils présentaient des granulations, parfois ils étaient disposés en chaînettes de trois à quatre unités. Au cours des périodes d'observation, les streptocoques disparurent, reparurent, se modifièrent, devinrent plus courts, la forme de palissade disparut, pour reparaître parfois et disparaître définitivement; parfois la faculté de prendre le Gram disparut et reparut ultérieurement. Dans un échantillon fut observée à certain moment 
la présence simultanée de streptocoques, prenant et ne prenant pas le Gram. Toujours l'apparition de streptocoques fut précédée de celle de bacilles en plus grand nombre, souvent en chaînettes, souvent à contours ondulés et à granulations ; tandis que durant la présence des streptocoques le nombre de bacilles diminua et que, lorsque les streptocoques devinrent plus courts ou disparurent, le nombre d'unités cocciformes isolées ou accouplées, ayant la même forme que ceux qui composaient les chaînettes streptococciques, augmenta.

A la fin des périodes d'observation, la flore bactérienne était composée de mierocoques prenant le Gram, isolés, en diplocoques ou en grappes, de différentes dimensions, de bacilles longs de 1 à $1,5 \mu$, larges de 0,5 à $0,6 \mu$, prenant ou ne prenant pas le Gram, à extrémités arrondies ou effilées, parfois incurvés, à granulations parfois multiples, parfois uniquement aux extrémités; parfois isolés, parfois disposés en séries de deux à trois unités; à flore bactérienne similaire à celle trouvée fréquemment dans des mamelles présentant des lésions de mammite chronique.

Il est intéressant de mentionner que Davis et Matrick isolèrent de fromage Cheddar, présentant des taches ayant une couleur de rouille, un bacille ayant les dimensions de $1,5-0,6 \mu$ à extrémités arrondies ou effilées, parfois ineurvé, prenant le Gram en cultures jeunes, tandis que dans les cultures vieilles peuvent se présenter des formes ne prenant pas le Gram ; organisme auquel ils reconnurent les caractéristiques d'une véritable bactérie lactique [16], et au sujet duquel ils mentionnent, qu'il est connu qu'au moins un organisme lactique (Streptococcus mastitidis) peut produire anaérobiquement et dans certaines conditions un pigment rouge [17].

Nombre de fois au cours de ces recherches fut donc constatée la présence, pendant et après le développement de streptocoques ayant provoqué vraisemblablement la mammite, des bacilles présentant les mêmes caractères morphologiques; et deux fois d'abord dans une culture dans du lait stérilisé de matériel prélevé d'une mamelle présentant des Jésions de mammite, ensuite dans du lait provenant d'une vache atteinte de mammite ; il y eut formation d'un pigment brunâtre, en présence de ces bacilles.

Davis et Matrick mentionnent encore que, pour la formation du pigment par le bacille, qu'ils isolèrent, il faut la présence d'un facteur, qu'ils ne parvinrent pas à identifier, présentant une certaine analogie avec la vitamine antinévritique ; facteur qui peut se trouver dans les tissus animaux et végétaux. Il est possible que dans les deux cultures, où se forma le pigment rouillé au cours de ces recherches, le facteur non identifié aurait pu se trouver: pour le premier cas, apporté par le matériel prélevé de la mamelle; pour le second cas, 
dans le lait même. Il importe cependant de signaler, que dans le second eas le pigment se forma dans une couche de flocons visqueux à la surface de l'échantillon, et que Davis et Matriok affirment que, dans les milieux de culture liquides, le pigment se trouve toujours dans le fond des récipients [18], ce qui eut lieu dans le premier cas.

Les données fournies par l'observation d'échantillons de lait de vaches atteintes de mammite furent done semblables à celles fournies par l'observation de cultures, dans du lait stérilisé, de matériel prélevé de mamelles, présentant des lésions de mammite, tant pour ce qui concerne les modifications d'aspect du lait, que celles de la flore bactérienne.

Le fait que, dans la grande majorité des cas, le matériel prélevé des mamelles présentant des lésions de mammite ne contient pas de streptocoques; que, pour ainsi dire, toujours, s'y trouvent des microcoques prenant le Gram, isolés, en diplocoques ou en grappes, de diverses dimensions; que, généralement, lorsque des cultures de ce matériel sont faites dans du lait, il y a dévéloppement de streptocoques, tandis que persistent des microcoques du même type que ceux trouvés dans la mamelle, et que dans six échantillors de lait, provenant de vaches atteintes de mammite, fut constatée la présence d'une flore bactérienne ayant des caractéristiques similaires, dont l'évolution fut identique, peut être considéré, à mon avis, comme indication que ces microcoques prenant le Gram ne seraient que des formes évolutives des streptocoques qui ont provoqué la mammite; ou bien que, parmi ces microcoques, se trouveraient des formes évolutives d'autres streptocoques que l'examen microscopique ne permet pas évidemment de distinguer des organismes ayant la même forme et que l'on considère généralemènt comme des saprophytes.

A ce sujet, il convient de rappeler les observations de Gorini concernant les coccus mammaires et celles de HUCKER concernant les cocci acido-protéolytiques.

Le fait que l'apparition de streptocoques fut généralement précédée de celle de bacilles, souvent disposés en chaînettes, présentant souvent des contours ondulés, des granulations et dont l'aspect permet de supposer un début de scission en organismes cocciformes, que ces bacilles étaient moins nombreux, ou avaient disparu lors de la présence des streptocoques et après leur disparition, alors quese trouvaient fréquemment, dans les cultures, de nombreux éléments cocciformes isolés ou accouplés, semblables à ceux qui formaient les chaînettes strèptococciques, tend à élayer l'hypothèse, émise par HuCKER et PEDERSON, que le genre "Streptococcus " serait intermédiaire, au point de vue morphologique, entre le type bacille et le type coccus. 


\section{CONCLUSIONS.}

Selon les données obtenues ici, la mammite de la vache serait provoquée, dans la très grande majorité des cas, par une infection streptococcique, ainsi que l'ont affirmé nombre d'auteurs.

L'existence de la mammite ne peut être établie que par l'examen d'échantillons de lait individuels et l'examen clinique de la vaché.

Les streptocoques trouvés dans les frottis du matériel prélevé sur des mamelles présentant des lésions de mammite, ceux qui se développèrent dans des cultures de ce matériel dans du lait stérilisé, ceux développés dans les six échantillons de lait provenant de vaches atteintes de mammite, n'avaient pas toujours la forme de palissade et ne prenaient pas toujours le Gram, données indiquant encore que ces earactéristiques ne peuvent être considérées comme indices de la faculté pathogène des streptocoques.

L'apparition des chaînettes streptococciques fut généralement précédée de l'apparition ou de la multiplication de bacilles, souvent disposés en chaînettes, présentant parfois un aspect pouvant laisser présumer un début de scission en unités cocciformes. Lorsque les chainettes streptococciques disparurent ou devinrent plus courtes, généralement fut constatée la présence, dans la culture, d'un plus grand nombre d'unités cocciformes isolées ou accouplées, ayant le même aspect que les unités dont étaient formées les chaînettes streptococciques. Cette constatation peut autoriser l'hypothèse que le streptocoque est l'intermédiaire entre le type bacillaire et le type cocciforme.

Lorsque des cultures, dans du lait stérilisé, furent faites de matériel prélevé sur des mamelles contenant en même temps des organismes lactiques et des bacilles de КосH, ces derniers disparurent avant ou lors de la coagulation provoquée par le développement des organismes lactiques. Fı́́quemment la mammite est constatée, lors de l'abattage, chez des vaches atteintes de tubereulose, et la présence du bacille de Kock dans la mamelle de ces vaches paraît plutôt rare. Au cours de ces recherches et de recherches antérieures [1], j'examinai les mamelles de 177 vaches, dont 147 étaient atteintes de tuberculose à divers degrés, et seulement 38 fois, je constatai la présence du bacille de KосH dans la mamelle.

Le fait que dans du lait stérilisé, ensemencé avec du matériel prélevé sur des mamelles contenant le bacille de КосH, celui-ci disparût avant ou lors de la coagulation, peut rendre admissible l'hypothèse, que si on trouve rarement ce bacille dans les mamelles de vaches atteintes de tuberculose, e'est qu'il ne parvient généralement pas à se fixer dans la mamelle que lorsque le tissu glandulaire est suffisamment altéré par la mammite pour que la sécrétion 
lactée soit tarie; que si avant ce stade, il pénètre dans le parenchyme mammaire, ce qui doit se produire fréquemment, puisque sa présence a été maintes fois constatée dans du lait, apparemment normal, prélevé, à la sortie de la mamelle, ehez des vaches atteintes de tuberculose, ne présentant pas de lésions de mammite décelables à l'examen clinique: il est évacué de la mamelle avec le lait, ou il y est détruit lorsqu'il y a rétention lactée.

La coexistence fréquente de la turberculose et de la mammite chez la varne, sans qu'il soit toujours possible de déceler le bacille de KосH dans la mamelle, indique, ainsi que j'en ai déjà exprimé l'avis antérieurement, que lorsqu'on constate la mammite chez la vache, elle est souvent la conséquence indirecte d'une affection générale qui, ayant débilité l'organisme, a facilité l'infection de la mamelle, et que, dans ces cas, il y a lieu de rechercher s'il n'y a pas simultanément l'existence de la tuberculose.

\section{BIBLIOGRAPHIE.}

[1] R. N. Göransson. Quelques observations sur la mammite de la vache. Le Lait, 1930, 10, 98, 974.

[2] C. Gorinr. Sur les eoccus mammaires (mammococcus) et les coecus analogues (caseococcus, enterococcus, gastrococcus). Le Lait, 1926, 6, 51, 61.

[3] C. Gorinr. Hétérogénéité et dissociation des coccus mammaires. Le Lait, $1930,10,97,745$.

[4] Hucker et Pederson. Studies on the Coccaceæ. XVI. The Genus Leueonostoc. New York Agricultural Experiment Station. Technical Bulletin, no 167 , juillet 1930 .

[5] G. J. Huckrer. Relationship of the various acid proteolytic cocci. Centralblatt für Bakteriologie, Parasitenkunde und Infektions krankhenite, 1926, 76.

[6] H. Kufferath. Sur les laits infectés par les streptocoques de la mammite des vaches laitières. Ann. de l'Institut Pasteur, 1921, 35, 167.

[7] A. A. Veenbaes. L'examen du dépôt dans le lait centrifugé, spécialement pour la recherche du lait streptococcique. Tijdschrift voor Diergeneeskunde, 1922, 49, 15. An. in Le Lait

[8] G. E. Jörgensen. La mammite parenchymateuse aiguë, avec références spéciales à l'étiologie et à la pathologie. Journal of the American Veterinary Association, $1924,64,6,699$.

[9] Skar. Mammites de la vache. Meddelse fra Staddyrlaegens lab. Norsk vet. tidsskrift, 1928, 30, 2, 3, 4. An. in Le Lait.

[10] Orla Jensen. Dairy Bacteriology (trad. Paul Arup). Londres, 1921.

[11] Ruнmekorf. Contribution à la question de la recherche des streptocoques dans le lait de marché. Zeitschrift für Fleisch- und Milchhygiene, 1924, 35, 6, 81. An. in Le Lait.

[12] C. F. VAN Oren. L'examen du sédiment du lait et l'importance de la recherche des streptocoques dans le lait. Het Algemmen Zuivelblad, 1922, 2, 28. Repris de Het Chemisch Weekblad. An. in Le Lait.

[13] A. A. Vennbas. L'examen du sédiment du lait et l'importance de la recher- 
che des streptocoques dans l'expertise du lait. Het Algemmen Zuivelblad. 1922, 2, 28. Repris de Het Chemisch Weekblad. An. in Le Lait.

[14] A. A. Veenbaas. L'examen du sédiment du lait, spécialement au point de vue de la signification des streptocoques dans l'appréciation du lait. Het Chemisch Weekblad, 1922, 19, 283. An. in Le Lait.

[15] C. Gorint. Action des streptocoques dans le lait. Comptes rendus de l'Académie des Sciences. An, in Le Lait.

[16] Davis et Matrick. Rusty Spot in Cheddar and other Cheese. I. Description of the causative organism. The Journal of Dairy Research. 1929, 1, 1, 50.

[17] Orla Jensen. The Lactic Acid Bacteria, 1919, Copenhague.

[18] Davis et Matrick. Rusty Spot in Cheddar and other cheese. II. Factors controlling the Pigmentation of the causative organism. The Journal of Dairy Research, 1930, 1, 2, 135 .

\section{LE FERMENT DE LA PRÉSURE ET LE LAIT (1)}

par

\section{RENÉ GALLAY}

Le phénomène banal, à portée de tout le monde, du lait qui tranche, qui caille, qui coagule sous l'action d'un peu de présure, a été l'objet de nombreuses recherches. Il existe sur cette question qui intéresse tout en même temps le physiologiste, puisque le phénomène se produit dans l'estomac et participe à la digestion, le chimiste, parce qu'il est important de savoir quelles sont les modifications moléculaires qui l'accompagnent, et le technicien, qui est forcé d'y voir la transformation du lait se trouvant à l'origine de l'industrie fromagère, une littérature volumineuse, encombrante, souvent touffue, regorgeant d'explications contradictoires, et dont la fantaisie n'est pas toujours exclue. Le but que nous nous sommes proposés aujourd'hui est de dégager de ce fatras de connaissances, de chiffres et d'hypothèses, les connaissances qui sont le plus solidement étayées sur l'expérimentation et, en nous aidant parfois d'observations et de recherehes personnelles, tenter de leur donner leur signification réelle, pour répondre finalement à ces trois questions : qu'est-ce que la présure ? quel est le mécanisme de son action chimique sur le lait ? et pourquoi conduit-elle à la prise en masse de ce liquide, e'est-à-dire à la solidification d'une partie de ses constituants ?

Le ferment soluble, l'enzyme de la présure, capable, sous certaines conditions, de trancher le lait, est produit par l'estomac de tous les mammifères, mais en quantité fort variable. On le connaît

(1) Leçon inaugurale d'un cours de chimie du lait, prononcée le 25 avril 1934 à l'Université de Lausanne. 